\title{
EXTREMAL ELEMENTS OF A CONVEX CONE OF SUBADDITIVE FUNCTIONS ${ }^{1}$
}

\author{
E. K. MCLACHLAN
}

1. Introduction. Let $E$ be an arbitrary set of elements, and let $\varrho$ be a class of subsets of $E$ such that (a) $\varnothing$, the empty set, and $E$

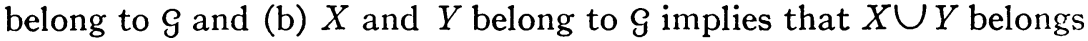
to $g$. Thus under the operation union $G$ is a commutative semi-group with a zero element. Let us partially order $\mathcal{G}$ by inclusion. Then the set of maps $f$ from $G$ into the real line such that for any $X$ and $Y$ belonging to $\mathrm{g}$ :

(i) $f(X) \geqq 0$,

(ii) $f(X) \leqq f(Y)$ when $X \subset Y$,

(iii) $f(X)+f(Y) \geqq f(X \cup Y)$,

form a convex cone $\mathfrak{e}$ (that is, $\mathfrak{e}+\mathcal{e} \subset \mathcal{e}$ and $\lambda \mathfrak{e} \subset \mathcal{e}$ for $\lambda>0$ ). When $\mathcal{G}$ is finite we shall give a characterization of the extremal elements of $\mathcal{e}$ and when $\mathcal{G}$ is not finite we shall give a characterization of a certain subcone of $\mathfrak{e}$. By definition a function $f \neq 0$ which belongs to $\mathfrak{e}$ is called an extremal element of $\mathfrak{e}$ if when $f=f_{1}+f_{2}$ for $f_{1}$ and $f_{2}$ belonging to $\mathfrak{e}$ there exist non-negative scalars $\lambda_{1}$ and $\lambda_{2}$ such that $f_{1}=\lambda_{1} f$ and $f_{2}=\lambda_{2} f$ (that is, $f$ has only proportional decompositions).

There are classes of set functions which form cones like $\mathfrak{e}$, for instance certain measures and capacities (see $[3 ; 1])$. The range space for the functions of $\mathfrak{C}$ could easily be a more general space and there are, of course, other interesting semi-groups on which the functions of $\mathfrak{e}$ could be defined. Choquet [1] has characterized the extremal elements of two cones closely related to $\mathfrak{C}$. One contains $\mathfrak{C}$ as a subcone and the other is contained in $\mathcal{C}$.

2. Preliminary theorem and definition. The following theorem will give us some general information on decomposing functions of $\mathfrak{C}$.

THEOREM 1. Let $f_{1}, f_{2}$, and $f$ belong to $\mathfrak{e}$ and let $f=f_{1}+f_{2}$. Then we have the following:

(i) If $f(X)=0$ for $X \in \mathcal{G}$, then $f_{1}(X)=f_{2}(X)=0$.

(ii) If $X$ and $Y$ are two elements of $\mathcal{G}$ such that $X \leqq Y$ (that is, $X \subset Y)$ and such that $f(X)=f(Y)$, then $f_{1}(X)=f_{1}(Y)$ and $f_{2}(X)=f_{2}(Y)$.

Presented to theSociety, December 27,1955; received by the editors March 7, 1960 .

1 This work which was supported by National Science Foundation grant NSFG1126 was presented to the Graduate School of the University of Kansas in partial fulfillment of the requirements for the degree of Doctor of Philosophy. 
(iii) If $X$ and $Y$ are two elements of $\mathcal{S}$ such that $f(X \cup Y)=f(X)$ $+f(Y)$, then $f_{1}(X \cup Y)=f_{1}(X)+f_{1}(Y)$ and $f_{2}(X \cup Y)=f_{2}(X)+f_{2}(Y)$.

Proof. The conclusion (i) follows from the fact that the elements of $\mathfrak{C}$ are non-negative. We know that the functions of $\mathfrak{e}$ are nondecreasing and hence we have $f_{1}(X) \leqq f_{1}(Y)$ and $f_{2}(X) \leqq f_{2}(Y)$. However, if $f_{1}(X)<f_{1}(Y)$, then $f(X)=f_{1}(X)+f_{2}(X)<f_{1}(Y)+f_{2}(Y)=f(Y)$. This contradicts the hypothesis that $f(X)=f(Y)$, and hence (ii) is true. Since $f_{1}$ and $f_{2}$ belong to $\mathcal{e}$ we have $f_{1}(X \cup Y) \leqq f_{1}(X)+f_{1}(Y)$ and $f_{2}(X \cup Y) \leqq f_{2}(X)+f_{2}(Y)$. If $f_{1}(X \cup Y)<f_{1}(X)+f_{1}(Y)$, then $f(X \cup Y)$ $=f_{1}(X \cup Y)+f_{2}(X \cup Y)<f_{1}(X)+f_{2}(X)+f_{1}(Y)+f_{2}(Y)=f(X)+f(Y)$. That is, $f(X \cup Y)<f(X)+f(Y)$, which contradicts the hypothesis.

In other words, this theorem says that on the sets of $\mathcal{G}$ where a function $f$ of $\mathfrak{C}$ is zero, constant, or additive, the decomposition functions must also be respectively zero, constant, or additive.

We shall now define an equivalence relation which is dependent upon an arbitrary element $f$ of $\mathfrak{e}$.

Definition. If $X$ and $Y$ belong to $\mathcal{G}$, then $X$ is $f$-equivalent to $Y$ if and only if there exists a finite sequence of elements, $X=X_{0}, X_{1}$, $\cdots, X_{k}=Y$ of $g$ such that $X_{i} \subset X_{i+1}$ or $X_{i} \supset X_{i+1}$ and $f\left(X_{i}\right)=f\left(X_{i+1}\right)$ for $i=0,1, \cdots, k-1$. The $f$-equivalence classes of the elements of $\mathcal{S}$ may be arranged in an indexed family $a_{\alpha}(f)$ where $\alpha \in A$. Let $\mathfrak{Q}_{\alpha}(f)$ be the $f$-equivalence class which contains the set $E$.

3. The main theorem. Notice first that each $f$ of $\mathfrak{e}$ is constant on each $f$-equivalence class $a_{\alpha}(f)$ and by Theorem 1 if $f_{1}$ and $f_{2}$ belong to $\mathcal{C}$ and $f=f_{1}+f_{2}$ then $f_{1}$ and $f_{2}$ must also be constant on each $\mathbb{Q}_{\alpha}(f)$.

Let $\mathfrak{C}^{*}$ be the subset of (normalized) functions $f$ of $\mathfrak{e}$ such that $f(E)=1$. Clearly, each function $f$ of $\mathcal{e}$ except $f \equiv 0$ is such that $f(E)>0$ and hence each function $f$ of $\mathcal{e}$ is a non-negative scalar multiple of an element of $\mathfrak{C}^{*}$. Then the extremal elements of $\mathfrak{C}$ are (strictly) positive scalar multiples of extremal elements of $\mathfrak{e}$ that belong to $\mathrm{e}^{*}$. Denote by $\bar{\lambda}_{\alpha}(f)$ the value of $f(X)$ for $X \in a_{\alpha}(f)\left(\bar{\lambda}_{\alpha_{0}}(f)=1\right)$ and let $\lambda_{\alpha}(f)$ be a variable that is associated with the number $\bar{\lambda}_{\alpha}(f)$ for $\alpha \neq \alpha_{0}$.

Let $\left\{X_{\beta}, Y_{\beta}\right\}$ where $\beta \in B$ be the set of all pairs of elements of $\mathcal{G}$ such that: $f\left(X_{\beta}\right)+f\left(Y_{\beta}\right)=f\left(X_{\beta} \cup Y_{\beta}\right)$. The set $B$ may be void. Let $S(f)$ be the system of equations thus obtained. In $S(f)$ substitute $\lambda_{\alpha}(f)$ in place of $f(X)$ when $X \in Q_{\alpha}(f)$ for $\alpha \neq \alpha_{0}$ and substitute one in place of $f(X)$ when $X \in Q_{\alpha_{0}}(f)$. Designate the system of equations $S(f)$ by $S^{\prime}(f)$ after the above substitutions have been made. Now $S^{\prime}(f)$ has a solution, namely $\lambda_{\alpha}(f)=\bar{\lambda}_{\alpha}(f)$ for $\alpha \neq \alpha_{0}$. 
If the number of equivalence classes of $f$ is finite, then we have the following:

Lemma 1. If $f \in \mathfrak{e}^{*}$ and $a_{1}(f), \cdots, a_{p}(f)$ are the $f$-equivalence classes where $E \in Q_{p}(f)$, if $\lambda_{i}(f)$ for $i=1, \cdots, p-1$ and 1 appears in $S^{\prime}(f)$, and if $\lambda_{i}(f)=\bar{\lambda}_{i}(f)$ for $i=1, \cdots, p-1$ is the only solution of $S^{\prime}(f)$, then $f$ is an extremal element of $\mathfrak{C}$.

Proof. Since $S^{\prime}(f)$ has a unique solution then $S^{\prime}(f)$ must consist of $p-1$ independent equations and hence $S^{\prime}(f)$ may be written in matrix form as follows: $\left[\alpha_{j i}\right]\left\{\lambda_{i}(f)\right\}=\left\{\gamma_{j}\right\}$ where $i$ and $j=1,2, \cdots$, $p-1$. Not all of the $\gamma_{j}=0$ since 1 appears in $S^{\prime}(f)$.

Let $f_{1}$ and $f_{2}$ be a decomposition of $f$. If $f_{i}(E)=0$ for $i=1$ or 2 then $f_{i} \equiv 0$. Hence let us take $f_{1}(E)=a, 0<a<1$, and then $f_{2}(E)=1-a$. By Theorem 1 the function $f_{1}$ (and $f_{2}$ ) must be additive on the pairs $\left\{X_{\beta}, Y_{\beta}\right\}$ where $\beta \in B$. Let us alter $S^{\prime}(f)$ by replacing 1 by $a$. Now the solution of $S^{\prime}(f)$ is $\lambda_{i}(f)=a \bar{\lambda}_{i}(f)$ for $i=1, \cdots, p-1$. Hence $f_{1}(X)$ must be equal to $a \bar{\lambda}_{i}(f)$ for $X \in Q_{i}(f), i=1, \cdots, p-1$. Therefore, $f_{1}$ (and $f_{2}$ ) is proportional to $f$.

If $B$ is void then there is at least one $\lambda_{i}(f), i=1, \cdots, p-1$ or 1 which fails to appear in $S^{\prime}(f)$.

Lemma 2. Let $f \in \mathfrak{C}^{*}$ and let $a_{1}(f), \cdots, a_{p}(f)$ be the f-equivalence classes where $E \in Q_{p}(f)$ if $\lambda_{i_{0}}, 1 \leqq i_{0} \leqq p$ does not appear in $S^{\prime}(f)$ and if $p>2$, then $f$ is not an extremal element of $\mathfrak{e}$.

Proof. Let $\delta_{1}=\min (f(Y)-f(X))$ for $X, Y \in \mathcal{G}, X \subset Y$ and $f(Y)$ $>f(X)$. Let $\delta_{2}=\min (f(X)+f(Y)-f(X \cup Y))$ for $X, Y \in \mathcal{G}$ and $f(X)$ $+f(Y)>f(X \cup Y)$. Lastly, let $\delta_{3}=\min (f(X), X \in \mathcal{G}, f(X)>0)$. Notice that $\lambda_{i_{0}}(f)>0$ for if $\lambda_{i_{0}}(f)=0$, then $\varnothing \in Q_{i_{0}}(f)$ and since $f(\varnothing)+f(X)$ $=f(X \cup \varnothing)$ for any $X \in \mathcal{G}$, we would have $\lambda_{i_{0}}(f)$ appearing in $S^{\prime}(f)$. Clearly, $\delta_{1}, \delta_{2}$, and $\delta_{3}$ are well-defined and since the range of $f$ is finite it follows that they are strictly positive. Take

$$
f_{1}(X)= \begin{cases}f(X) / 2, & X \notin \mathfrak{Q}_{i_{0}}(f), \\ (f(X) / 2)-\epsilon, & X \in \mathfrak{a}_{i_{0}}(f),\end{cases}
$$

and $f_{2}=f-f_{1}$ where $\epsilon=(1 / 8) \min \left(\delta_{1}, \delta_{2}, \delta_{3}\right)$. Thus $f_{1}$ and $f_{2} \in \mathcal{C}$, and $f_{1}$ and $f_{2}$ are not proportional to $f$.

If $p=1$ (that is, $f \equiv 1$ ) then it is easy to show that $f$ is an extremal element of $\mathfrak{C}$ (see Theorem 1). If $p=2$, then $0 \leqq \bar{\lambda}_{1}(f)<1$. If $\bar{\lambda}_{1}(f)=0$ then again by Theorem 1 we may easily see that $f$ is an extremal element of $\mathfrak{C}$. In case $\bar{\lambda}_{1}(f)>0$ then $f$ is an extremal element of $\mathfrak{e}$ if both $\lambda_{1}(f)$ and 1 appear in $S^{\prime}(f)$ (see Lemma 1), but if either $\lambda_{1}(f)$ 
or 1 fail to appear in $S^{\prime}(f)$ then the argument of Lemma 2 may be used to show that $f$ is not an extremal element of $\mathfrak{e}$.

It remains to determine what happens when each of the $\lambda_{i}(f)$, $i=1, \cdots, p-1$ and 1 appears in the system $S^{\prime}(f)$ and when $S^{\prime}(f)$ does not have a unique solution.

Lemma 3. Let $f \in \mathrm{e}^{*}$ and let $a_{1}(f), \cdots, a_{p}(f)$ be the f-equivalence classes where $E \in Q_{p}(f)$, if each $\lambda_{i}(f), i=1, \cdots, p-1$ and 1 appears in $S^{\prime}(f)$, and if $S^{\prime}(f)$ does not have a unique solution, then $f$ is not an extremal element of $\mathfrak{C}$.

Proof. Since 1 appears in $S^{\prime}(f)$, at least one equation in $S^{\prime}(f)$ is not homogeneous. Geometrically, each of the equations in $S^{\prime}(f)$ represents a hyperplane in $E^{p-1}$ space. All of these hyperplanes have a point in common since $\lambda_{i}(f)=\bar{\lambda}_{i}(f), i=1, \cdots, p-1$ is a solution of $S^{\prime}(f)$. Since the solution of $S^{\prime}(f)$ is not unique, then all of the hyperplanes of $S^{\prime}(f)$ meet in a linear variety $L$ which is more than just a point. At least one of the equations of $S^{\prime}(f)$ is not homogeneous and hence $L$ does not pass through the origin. Let $K$ be the open sphere in $E^{p-1}$ of center $\left(\bar{\lambda}_{1}(f), \cdots, \bar{\lambda}_{p-1}(f)\right)$ and of radius $\epsilon=(1 / 8)$ min $\left(\delta_{1}, \delta_{2}, \delta_{3}\right)$ (see the proof of Lemma 2). Let $L^{\prime}$ be the linear subspace of $E^{p-1}$ spanned by the point $\left(\bar{\lambda}_{1}(f), \cdots, \bar{\lambda}_{p-1}(f)\right)$. Then the set $(K \cap L)-L^{\prime}$ is nonvoid and the coordinates of each point of $(K \cap L)-L^{\prime}$ gives a solution of $S^{\prime}(f)$ which is not proportional to $\left(\bar{\lambda}_{1}(f), \cdots, \bar{\lambda}_{p-1}(f)\right)$. Let $\lambda^{\prime}=\left(\lambda_{1}^{\prime}(f), \cdots, \lambda_{p-1}^{\prime}(f)\right)$ belong to $(K \cap L)$ $-L^{\prime}$ and let $f_{1}(X)=\lambda_{i}^{\prime}(f) / 2$ when $X \in Q_{i}(f)$ where $\lambda_{p}^{\prime}(f)=1$. Then take $f_{2}(X)=f(X)-f_{1}(X)$. Thus since $\lambda^{\prime} \in K, f_{1}$ and $f_{2}$ belong to $\mathcal{C}$. Since $\lambda^{\prime} \in L^{\prime}, f_{1}$ and $f_{2}$ provide a nonproportional decomposition of $f$.

Upon the bases of the Lemmas 1, 2, and 3 we have the following theorem:

Theorem 2. Let $f \in \mathrm{e}^{*}, f \not \equiv 0$ and let $a_{1}(f), \cdots, a_{p}(f)$ be the $f$ equivalence classes where $E \in a_{p}(f)$. If $p=1$, then $f$ is an extremal element if and only if $f=1$. If $p=2$, then $f$ is an extremal element if and only if the range of $f$ is 0 and 1 or if both $\lambda_{1}(f)$ and 1 appear in $S^{\prime}(f)$ and $S^{\prime}(f)$ has a unique solution. If $p>2$, then $f$ is an extremal element if and only if each $\lambda_{i}(f), i=1, \cdots, p-1$ and 1 appears in $S^{\prime}(f)$ and $S^{\prime}(f)$ has a unique solution.

Thus the above theorem characterizes the extremal elements of the subcone $\mathfrak{C}^{\prime}$ of $\mathfrak{C}$ which consists of all of those functions of $\mathfrak{C}$ which have only a finite number of range values. The number of range values of $f$ cannot exceed the number of $f$-equivalence classes. Each 
extremal function of this subcone $\mathfrak{C}^{\prime}$ is an extremal function of $\mathfrak{C}$. It would be interesting to know if there are any extremal elements of $\mathfrak{C}$ that are not in $\mathfrak{C}^{\prime}$. If $\mathcal{G}$ is finite then $\mathfrak{e}^{\prime} \equiv \mathfrak{C}$. Whereas this characterization does give a method of easily determining if a given function of the cone is an extremal function or not, it does not indicate a procedure by which a complete list of extremal elements could be constructed. However, two things can be noted that would be helpful if a listing of the extremal functions is desired.

If $f$ is an extremal element of $\mathfrak{e}, f \in \mathfrak{e}^{*}$, and 0 belongs to the range of $f$ then $f(\varnothing)=0$ since $f$ is nondecreasing. If $X$ and $Y \in \mathcal{G}, f(X)=0$ and $f(Y)=0$ then $X$ and $Y$ are $f$-equivalent to $\varnothing$ and hence $X$ and $Y$ are $f$-equivalent. Thus let $a_{0}(f)$ be the $f$-equivalence class of all $X \in \mathcal{G}$ such that $f(X)=0$. Then since $f$ is an extremal element $\lambda_{0}(f)$ appears in $S^{\prime}(f)$. There exists $X_{\beta} \in Q_{0}(f)$ with $\beta \in B$ such that $f\left(X_{\beta}\right)+f\left(Y_{\beta}\right)=f\left(Y_{\beta}\right)=f\left(X_{\beta} \cup Y_{\beta}\right)$. Then $Y_{\beta}$ and $X_{\beta} \cup Y_{\beta}$ are $f$-equivalent and the equation appearing in $S^{\prime}(f)$ involving $\lambda_{0}(f)$ must be exactly $\lambda_{0}(f)=0$. For $f$ is an extremal function, $S^{\prime}(f)$ must have a unique solution and hence $S^{\prime}(f)$ must still have a unique solution with the equation $\lambda_{0}(f)=0$ missing. This means that to list the extremal elements of $\mathfrak{e}$ it is first desirable to list all of the possible classes of subsets of $\mathcal{G}$ on which $f$ of $\mathfrak{e}^{*}$ can be zero. Call these classes null classes. This means also that we may consider separately how $f$, an extremal function of $\mathfrak{e}$, behaves on complements of these null classes.

Suppose $Q_{1}(f), \cdots, Q_{p}(f)$ are the $f$-equivalence classes on which $f$, an extremal function of $\mathfrak{C}$, is nonzero, where $E \in a_{p}(f)$. If $X_{1}, \cdots, X_{p}$ of $g$ belong to the same $f$-equivalence class, if $X_{1} \subset X_{1}$ $\cup X_{2} \subset \cdots \subset\left\{\cup X_{i}, i=1, \cdots, p\right\}$ properly where $X_{1} \cup \cdots \cup X_{k}$ $\in a_{k}(f)$, for $k=1, \cdots, p$ and if $f\left(X_{i}\right)=1 / p, i=1, \cdots, p$, then from the subadditivity inequalities

$$
\begin{aligned}
2 / p & =f\left(X_{1}\right)+f\left(X_{2}\right) \geqq f\left(X_{1} \cup X_{2}\right), \\
3 / p & \geqq f\left(X_{1}\right)+f\left(X_{1} \cup X_{2}\right) \geqq f\left(X_{1} \cup X_{2} \cup X_{3}\right), \\
\text {. . . . . . . . . . . . . }, & \\
p(1 / p) & \geqq f\left(X_{1}\right)+f\left(X_{1} \cup \ldots \cup X_{p-1}\right) \geqq f\left(X_{1} \cup \ldots \cup X_{p}\right)=1,
\end{aligned}
$$

we get $f\left(X_{1} \cup \cdots \cup X_{k}\right)=k / p$ for $k=1, \cdots, p$. This gives one way for an extremal function to behave on the complements of the null classes of g. It would be interesting to know if this is the only way.

The interest in knowing the extremal elements of $\mathfrak{e}$ comes from the fact that it is possible under certain circumstances to give integral 
representations of all the elements of $\mathfrak{e}^{\prime}$ in terms of the extremal elements of $\mathfrak{e}^{\prime}$ (see [1]). In particular if $\mathfrak{e}^{*}$ is compact in the vector space $\mathfrak{e}^{\prime}-\mathfrak{e}^{\prime}$ (the smallest vector space containing $\mathfrak{e}^{\prime}$ ) with respect to a topology on $\mathfrak{e}^{\prime}-\mathfrak{e}^{\prime}$ that is compatible with the structure of the vector space then an integral representation exists. We have these conditions met if we use here the topology of simple convergence.

There are classes of set functions which form cones like $\mathfrak{e}^{\prime}$; for instance the set of outer measures defined on a finite hereditary ring (see [3]) and the set of capacities defined on the compacts of a finite topological space (see [1]).

4. Remarks. Let $g=g_{n}$ where $g_{n}$ consists of the mutually disjoint sets $\varnothing, A_{1}, \cdots, A_{n},(n \geqq 1)$ and all possible unions of these sets (this is the case where $\mathcal{G}$ is finite) and let $\mathfrak{C}_{n}$ be the cone $\mathfrak{C}$ associated with $g_{n}$. If $f \in \mathbb{C}_{n}$ then $f$ may have $p f$-equivalence classes where $1 \leqq p \leqq 2^{n}$. Since $g$ is finite, and since there are only a finite number of systems of equations $S(f)$ that can occur for a fixed $p$, Theorem 2 allows us to write out all of the extremal elements associated with a given pair of integers $p$ and $n$.

If $n=1, p=1$, then the only extremal elements of $\mathfrak{C}_{1}$ belonging to $\mathrm{C}_{1}^{*}$ is $f=1$. If $n=1$ and $p=2$, then $f(\varnothing)=0$ and $f\left(A_{1}\right)=1$ is a normalized extremal element of $\mathfrak{C}_{1}$. Since for $p=2$ there is no way to make both $\lambda_{1}(f)$ and 1 appear in $S^{\prime}(f)$ with $\bar{\lambda}_{1}(f)>0$ it follows that we now have all of the normalized extremal elements for $n=1$.

For $n=2$ let us exhibit $g_{2}$ and $f$ as follows:

$$
\mathrm{S}_{2}: \varnothing A_{A_{2}} A_{1} \cup A_{2}=E, \quad f: a_{0}{ }_{a_{2}}^{A_{1}} 1 .
$$

Then we shall understand by this that $f(\varnothing)=a_{0}, f\left(A_{1}\right)=a_{1}, f\left(A_{2}\right)=a_{2}$, and $f(E)=1$. The normalized extremal elements of $\mathfrak{C}_{2}$, within a permutation of the indices, are as follows:

1

(1) 01 ,

0
1

(2) $0 \quad 1$,

1
1

(3) 11 ,

1
.5

(4) $\quad .5 \quad 1$. .5

Using an analogous method for representing the normalized extremal elements for $n=3$, we have 


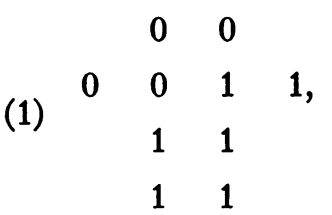
$0 \quad 1$
(2) $\begin{array}{llll}0 & 1 & 1 & 1 \text {, } \\ & & 1 & 1\end{array}$
(3) $0 \begin{array}{llll}0 & 1 & 1 & 1\end{array}$
(4) $\begin{array}{llll}1 & 1 & 1 & 1 \text {, } \\ & & 1 & 1\end{array}$
$\begin{array}{lllll}(5) & .5 & .5 & 1 & 1 \\ & & 1 & 1 & \end{array}$
$\begin{array}{lllll}\text { (6) } & 0 & .5 & 1 & 1 \text {, }\end{array}$
11 .51
$\begin{array}{lllll} & & .5 & 1 & \\ & & & & \\ & .5 & .5 & 1 & 1 \\ & & .5 & 1 & \end{array}$
$.5 \quad .5$
$\begin{array}{lllll}(8) & .5 & .5 & 1 & 1 \text {, }\end{array}$
.51
$.5 \quad .5$
$\begin{array}{lllll}\text { (9) } & 0 & .5 & .5 & 1 \text {, }\end{array}$
$\begin{array}{ccccc} & & .5 & .5 & \\ \text { (10) } & .5 & .5 & .5 & 1 \\ & & .5 & 1 & \end{array}$
$.5 \quad .5$
.51
$\begin{array}{lllll}(11) & .5 & .5 & .5 & 1 \text {, }\end{array}$
$.5 \quad .5$
$\begin{array}{ll}.5 & .5\end{array}$
$\begin{array}{lllll}(12) & 0 & .5 & .5 & 1\end{array}$
$1 / 3 \quad 2 / 3$
(13) $1 / 3 \quad 1 / 3 \quad 2 / 3 \quad 1$.
$1 / 3 \quad 2 / 3$

\section{REFERENCES}

1. G. Choquet, Theory of capacities, Technical Note No. 1, University of Kansas, Lawrence, Kansas, 1954.

2. - Theory of capacities, Ann. Inst. Fourier, Grenoble (1953-1954), pp. 131-295, 1955 (Papers [1] and [2] are identical).

3. P. R. Halmos, Measure theory, New York, Van Nostrand, 1950.

\section{UNIVERSITY OF KANSAS}

